\title{
Use of pericardial baffle in the management of intractable bleeding in patients undergoing aortic surgery
}

\section{Introduction}

Intractable bleeding can occur in complex aortic surgeries such as redo aortic surgeries, aortic dissection repair, endocarditis with root abscess and aortic arch repairs. The cause of such bleeding can be multi-factorial, which includes long bypass time, technique error, coagulopathy, fragile tissue etc. When common practice using reinforce sutures, local packing, massive transfusion of blood and blood products along with clotting agents fails, a pericardial baffle (modified Cabrol patch) may be the only option to save the patient.

We present three cases which intractable bleeding occurred during complex aortic surgeries and they were successfully managed with a pericardial baffle in two of them. Baffle technique is creating a peri-aortic space and shunting it into the right atrium. Need for multiple units of blood and blood products transfusions and longtime hemodynamic instability leads to immunosuppression increasing the risk of infection as well as the rise of morbidity and mortality.

We believe early decision to use baffle technique is the key to success. Certain complex aortic reconstructive procedures with a high bleeding rate which cannot be easily controlled by routine means including packing with sponges, topical hemostatic agents, blood factors and even in our cases using activated Factor VIII, could be successfully controlled with bovine pericardial baffle.

\section{Case \#1}

The first patient is a 64-year-old white gentleman with heavy drinking history and active immunosuppressive medication for psoriasis seborrheic dermatitis. In 2015, he underwent aortic valve replacement with a $25 \mathrm{~mm}$ Magna
Ease bioprosthetic valve for bicuspid aortic valve stenosis and the ascending aorta was replaced with a $32 \mathrm{~mm}$ Hemashield Dacron tube graft. The patient did well until March 2018 when he presented with acute bioprosthetic endocarditis with two cerebrovascular accidents. After six weeks of IV antibiotics, a transesophageal echocardiogram revealed the bioprosthetic valve was rocking with severe paravalvular regurgitation.

The patient underwent a redo mediastinotomy with femoral arterial and vein cannulation. There were very dense adhesions everywhere. After clamping aortic tube graft, cardioplegia was delivered through the retrograde cannula into the coronary sinus. Dacron tube graft was incised. The valve practically was hanging with a few sutures which were cut and the valve was removed. An outpouching under the left main coronary and a windsac in the noncoronary sinus towards the left atrium were present. After debridement of the aortic annulus, the windsac was removed and repaired. A 29 $\mathrm{mm}$ Medtronic Freestyle valve was placed in a subcoronary manner with 4-0 Prolene suture. Along with this, the outpouching under the left main and windsac was closed off. Following the closure of the aortotomy, cross clamp was removed. The heart recovered to the normal rhythm. The patient was successfully weaned off cardiopulmonary bypass. After protamine was given, he was bleeding from the aortic suture line due to fragile tissue. Multiple pledgeted sutures were placed along with administration of massive transfusion of the blood and blood products including platelets, coagulation factors including FFP cryoprecipitate and factor VII without success. At this point, we felt the only way to save his life was to create a periaortic space and connect this space to the right atrium to shunt the shed blood back into systemic

\section{Aryana Nazem*, Zhandong Zhou, and Charles Lutz}

Division of Cardiac Surgery, St. Joseph's Hospital, Syracuse, New York

*Author for correspondence:

17anazem@gmail.com 
circulation. Using a 4-0 prolene, a large piece of bovine pericardium was sutured to the pulmonary artery, right ventricular outflow tract, right atrial and superior vena cava to close off the space around the aorta as the transverse sinus was scared in. A $10 \mathrm{~mm}$ Hemashield Dacron graft was anastomosed to the middle of the pericardial baffle using a 5-0 prolene suture and the other end was anastomosed to the right atrium. The surgical field immediately becomes dry and we were able to close the chest (FIGURE 1). The patient was extubated six hours later and transferred to the cardiac floor the next day. The patient was discharged on post-op day 5 . His TEE revealed no shunting on discharge.

\section{Case \#2}

78-year-old gentleman on chronic steroid therapy presented with acute onset of chest pain. CT angiogram show acute type A aortic dissection. He was taken to the operating room immediately for surgical repair. Surgery was performed through a median sternotomy, cardiopulmonary bypass was established with femoral arterial and right atrial cannulation. The mid ascending aorta was initially clamped and the cardioplegia was given retrogradely. After opening the ascending aorta, we noticed tissue quality was very poor. The intimal tear extended to the aortic arch. Therefore a full root Bentall procedure with hemi-arch replacement was performed using a $21 \mathrm{~mm}$ Freestyle Porcine root. Re-implantation of the coronaries was done. Since the dissection was extended to the arche, the patient was cooled to 20-degree centigrade and then circulatory arrest was achieved, hemiarch replacement was performed. After cross-clamp was removed, the distal anastomosis started bleeding. After multiple pledgeted suture repair and massive transfusion of blood and blood products, the bleeding did not subside. The decision was made to create a peri-aortic space to right atrium shunt. A 4-0 prolene suture was used to close transverse sinus first. Then a large piece of the pericardium was used to contain the area around the aorta and a $10 \mathrm{~mm}$ Dacron graft was used to connect the middle of the pericardium to the right atrium in the same fashion as described in the first case. The surgical field almost immediately dried up. The patient's postoperative course was uneventful. His CT angiogram revealed small patent shunt. He feels well and does not wish to have any more intervention.

\section{Case \#3}

A 45-year-old gentleman with a history of chronic renal disease and thirty years of smoking, morbid obesity who presented to emergency room with sudden onset of chest pain for six hours. CT scan revealed acute type A dissection of the aorta extending from ascending into the abdominal aorta. He was anuric and hypotensive. The patient was immediately taken to the operating room on multiple vasopressors. After heparinization, right femoral artery and vein were cannulated.

Upon entering the pericardium, clots and blood gushed out and blood pressure recovered, which was suggestive of tamponade. Cardiopulmonary bypass started, and the patient was cooled down to 20-degree centigrade with a left ventricular vent in place. Cross-clamp was applied and retrograde cardioplegia was given. A composite graft was used with re-implantation of the coronaries. The extent of the dissection had practically denuded the adventitia from the coronary buttons, but they were re-implanted successfully. The distal section of the heatshield graft was anastomosed to the beginning of the arch under hypothermic circulatory arrest after it was fortified by applying Bioglue. Patient's tissue was poor. After cross-clamp was removed we had to clamp a few times to place repairing sutures. The right coronary attachment fell apart due to tissue quality which necessitated right coronary ligation and bypassing right coronary artery with a reversed saphenous vein graft. At this point, we had six hours of cardiopulmonary bypass time and after coming off cardiopulmonary bypass and massive transfusion of blood and blood products the patient still had remarkable bleeding. Blood pressure was maintained and the baffle procedure dried up the field. However, the patient did not do well due to profound shock and acidosis when the family made the patient DNR and ultimately the patient succumbed to death.

\section{Discussion}

Uncontrollable bleeding in complex aortic surgery is not uncommon. It usually occurs in complex cases with poor tissue quality, long cardiopulmonary bypass time and hypothermic circulatory arrest cases. These conditions may be associated with catastrophic coagulopathy. In complex aortic reconstruction cases, the 


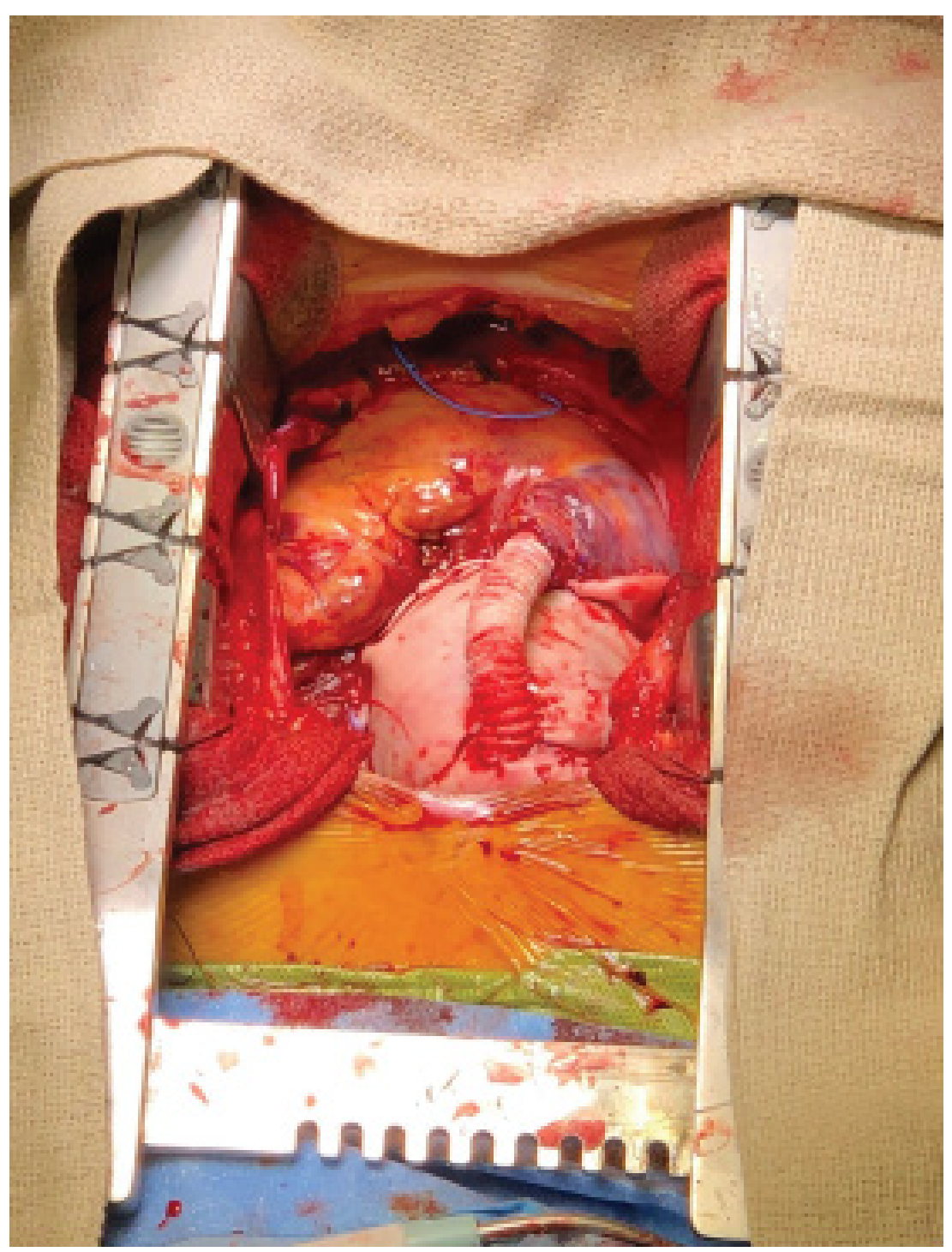

FIGURE 1. Case report\#1.

cardiopulmonary bypass time is already long and second pump run for reinforcing the suture line may carry higher morbidity and mortality [1].

If a discrete bleeding site could not be found or suturing is not working, baffling makes practical sense. Such serious bleeding does not respond to ordinary measures and ultimately carries a very high mortality and morbidity. Perigraft to right atrium shunt was first described by Cabrol and colleagues [2]. Since then many modifications have been described by Thomas A Salerno and colleagues [3], John A Elefteriades [4], Hoover and colleagues [5] and Marc Blum [6].

In re-do cases or acute dissection of ascending aorta, the rate of serious bleeding is high, therefore some surgeons use Cabrol fistula in a higher percentage of this kind of cases [7].
Perigraft to right atrial fistula makes seemingly uncontrolled bleeding field into a suddenly dry one. Moreover, it makes the closure of the chest possible. Surprisingly the complications of the baffle procedure are rare or negligible. Majority of fistulas close in hours or days after surgery.

It is important to identify patients who are undergoing aortic reconstruction and are at higher risk for intractable bleeding [8]. The basic technique for the creation of baffle is by sewing an autologous or bovine pericardium to seal the space around the aorta. To do that it is advised to close the transverse sinus at the onset. It is done by approximation of the right pulmonary artery to the dome of the left atrium ideally before the aortic reconstruction is started. It would be difficult to do it once the reconstruction is completed. In our cases, the baffle was not intended at the beginning but it was done at the end for salvaging the patients. The purpose of the baffle creation is to let clot seal most of the bleeding sites and shunt the shed blood back into systemic circulation, therefore, diminishes blood transfusion and its associated complications such as bacterial endocarditis [9], prolonged ventilation, and other morbidities and mortalities [10].

Short and long term side effects of this procedure are early clotting of the shunt or persistent shunting. In the majority of the cases, shunts will clot. The ones with persistent shunting are usually asymptomatic and it is rare for such patients to need reoperation. In our cases only one had persistent but it was an asymptomatic shunt.

Cabrol [11] reported a series of 260 patients who had a prophylactic shunt, only three cases had persistent flow. Two of the three needed reoperation. Hoover and colleagues [12]. reported nine cases of shunt creation without any death. Two had a patent shunt and one needed reoperation. In our series, we closed off the space surrounding the aorta by suturing bovine pericardium over these structures and connect the contained peri-aortic space to right atrial appendage. This technique is what has been described by Posacuglu and colleagues [13]. In seven cases who underwent type $A$ aortic dissection repair, they used autologous or bovine pericardium. In his series, there was no patent shunt on discharge TEE or CT scan.

Complications of this procedure are early 
thrombosis, bleeding, persistent patent shunt. Right heart failure is a theoretic concern and there are no reported cases of reoperation for that reason. In Toole's [8]. series there were six patients with persistent fistulas but only one needed re-intervention.

Baffle procedures save lives since mortality in this subset of patients is very high. Toole and colleagues [8]. reported their observed mortality to be $4 \%$ in the baffled group compared with the group that underwent aortic surgery. In their series, they were able to close the chest in $74 \%$ of the patients in the baffled group. The baffle group had a higher rate of prolonged ventilation while the incidence of postoperative renal failure, stroke, reoperation for bleeding and mediastinitis was similar. It is our observation that baffle procedure has saved two of our cases who otherwise would have died. In our third case who did not survive, we should have decided earlier to do the baffle to prevent blood loss and ultimate the demise of the patient. Baffle not only decreases mortality, but it also cuts down on morbidity associated with massive transfusion. It is our belief that baffle converts possible devastating postoperative course and outcome to a more normal one.

In a subset of patients with very high-risk aortic reconstruction, mortality was estimated in the range of $22 \%$ to $57 \%$. Tools and colleagues reported only $4 \%$ mortality in this high-risk group since they used the baffle [8]. Their mortality in cases who did not need baffle was $6 \%$ which suggest baffle technique may significantly reduce the mortality in high-risk aortic surgeries.

\section{Conclusion}

The use of pericardial peri-aortic baffle in patients with intractable bleeding may be lifesaving. The baffle is a vehicle for autotransfusion of shed blood preventing complications associated with hemodynamic instability and multiple transfusion. Early use the baffle technique in high-risk aortic surgeries may decrease mortality and morbidity to normal levels. It may be wise to anticipate severe postcardiopulmonary bypass bleeding at the onset of some high-risk aortic surgeries and consider the pericardial baffle as a preventative technique. 


\section{References}

Haken Posacioglu, Anil Ziya Apadyin, Tahir Yagdi, Faith Islamoglu. Perigraft to atrial shunt by using autologous pericardium for control of bleeding in acute Type A dissection. Ann. Thorac. Surgery 74, 1071-1074 (2002).

Cabrol C, Parie A, Gandjbakch I, et al. complete replacement of ascending aorta with reimplantation of coronary arteries: a new Surgical Approach. J. Thorac. Cardiovasc. Surg. 81(2), 209-215 (1981).

Salerno TA, Carvalho EM, Panos AL, Ricci M. Modified cabrol shunt after complex aortic surgery. Ann. Thorac. Surgery. 86(2), 669-670 (2008).

Elefteriades JA, Youssef S, Rousou L, Nazer R. Novel valved graft modification of cabrol fistula for bleeding after aortic roots. Ann. Thorac. Surg. 94(5), 17411743 (2012).

Hoover EL, Hsu HK, Ergin A, et al. Left to right shunts in control of bleeding following surgery for aneurysm of the ascending aorta. Chest. 91, 844-849 (1987).

Blum M, Panos A, Lichtenstein SV, Salerno TA. Modification cabrol shunt for control of hemorrhage in repair of Type A dissection of the aorta. Ann. Thorac. Surg. 48(5), 709-711 (1989).

Estrera AL, Miller CC, Kaneko T, et al. Outcomes of acute Type A aortic dissection after previous cardiac surgery. Ann. Thorac. Surg. 89(5), 1467-1474 (2010).

Toole JM, Stroud MR, Ikonomidis JS. Salvage periaortic pericardial baffle equalizes mortality in bleeding patients undergoing aortic surgery. J. Thorac. Cardiovasc. Surg. 148(1), 151-155 (2014).

Chelemer SB, Prato BS, Cox PM Jr, O'Connor GT, Morton JR. Association of bacterial infection and red blood cell transfusion after coronary artery bypass surgery. Ann. Thorac. Surg. 73(1), 138142 (2002).

Koch CG, Li L, Duncan AI, et al. Morbidity and mortality risk associated with red blood cell and blood-component transfusion in isolated coronary artery bypass grafting. Crit. Care Med. 34(6), 1608-1616 (2006).

Cabrol C, Cush EM. Shunt control of bleeding after homograft replacement of the ascending aorta. Ann. Thorac. Surg. 67(4), 1162-1163 (1999).

Hoover EL, Hsu HK, Ergin A, et al. Left to right shunt in control of bleeding following surgery for aneurysm of ascending aorta. Chest. 91(6), 844-849 (1987).

Posacioglu H, Apaydin AZ, Calkavur $T$, et al. Perigraft to right atrial shunt by using autologous pericardium for control of bleeding in acute type A dissections. Ann. Thorac. Surg. 74(4), 1071-1074. 